\title{
Prescription of Kampo Drugs in the Japanese Health Care Insurance Program
}

\author{
Kotoe Katayama, ${ }^{1}$ Tetsuhiro Yoshino, ${ }^{2}$ Kaori Munakata, ${ }^{2}$ Rui Yamaguchi, ${ }^{1}$ Seiya Imoto, ${ }^{1}$ \\ Satoru Miyano, ${ }^{1}$ and Kenji Watanabe ${ }^{2,3}$ \\ ${ }^{1}$ Human Genome Center, Institute of Medical Science, University of Tokyo, 4-6-1 Shirokanedai, Minato-ku, Tokyo 108-8639, Japan \\ ${ }^{2}$ Center for Kampo Medicine, Keio University School of Medicine, 35 Shinano-machi, Shinjuku-ku, Tokyo 160-8582, Japan \\ ${ }^{3}$ Faculty of Environment and Information Study, Keio University, 5322 Endo, Fujisawa, Kanagawa 252-0882, Japan \\ Correspondence should be addressed to Kenji Watanabe; watanabekenji@a6.keio.jp
}

Received 29 June 2013; Revised 25 September 2013; Accepted 8 October 2013

Academic Editor: Heidrun Reissenweber-Hewel

Copyright (C) 2013 Kotoe Katayama et al. This is an open access article distributed under the Creative Commons Attribution License, which permits unrestricted use, distribution, and reproduction in any medium, provided the original work is properly cited.

\begin{abstract}
Kampo medicine or traditional Japanese medicine has been used under Japan's National Health Insurance scheme for 46 years. Recent research has shown that more than $80 \%$ of physicians use Kampo in daily practice. However, the use of Kampo from the patient perspective has received scant attention. To assess the current use of Kampo drugs in the National Health Insurance Program, we analysed a total of 67,113,579 health care claim records, which had been collected by Japan's Ministry of Health, Labour and Welfare in 2009. We found that Kampo drugs were prescribed for $1.34 \%$ of all patients. Among these, $92.2 \%$ simultaneously received biomedical drugs. Shakuyakukanzoto was the most frequently prescribed Kampo drug. The usage of frequently prescribed Kampo drugs differed between the youth and the elderly, males and females, and inpatients and outpatients. Kampo medicine has been employed in a wide variety of conditions, but the prescription rate was highest for disorders associated with pregnancy, childbirth, and the puerperium (4.08\%). Although the adoption of Kampo medicine by physicians is large in a variety of diseases, the prescription rate of Kampo drugs is very limited.
\end{abstract}

\section{Introduction}

Interest in traditional medicine has increased globally in recent years. The World Health Organization (WHO) recommends that physicians use traditional medicine for care and treatment and encourages the integration of traditional medicine into the next edition of the International Statistical Classification of Diseases and Related Health Problems (ICD11) $[1-4]$.

Kampo medicine (Japanese traditional medicine) became integrated into the Japanese health care system - the National Health Insurance Program - 46 years ago alongside modern medicine, and the program has covered all citizens since 1961.

Because there is only one type of medical license in Japan, physicians prescribe both Kampo medicine and western biomedicine. At present, 148 Kampo extracts are approved as prescription drugs. Currently, $80-90 \%$ of physicians use Kampo drugs in daily practice [5-9], but most use a very limited number of Kampo drugs. In that sense, the number of patients receiving prescriptions for Kampo drugs is unknown. Similarly, their joint use with biomedical drugs has not received the appropriate amount of attention. In this study, therefore, we examine Kampo drug prescription within the Japanese national health care system.

\section{Material and Method}

The Ministry of Health, Labour and Welfare (MHLW) of Japan collects health care claims data annually. The MHLW conducts an annual survey in order to assess the medical status of treatments, diseases and injuries, and drug administration; obtain basic data for the management of medical insurance; and report on the use of drugs under the health insurance system managed by the Japan Health Insurance Association, society-managed employment-based 
TABLE 1: Numbers of institutions and detailed statements reviewed in the surveillance study.

\begin{tabular}{|c|c|c|c|c|}
\hline & \multirow{2}{*}{$\begin{array}{l}\text { Number of medical } \\
\text { institutions }\end{array}$} & \multicolumn{3}{|c|}{ Number of detailed statements } \\
\hline & & Total & $\begin{array}{c}\text { General medical } \\
\text { care }\end{array}$ & $\begin{array}{l}\text { Medical care system for } \\
\text { the elderly in the later } \\
\text { stages of life }\end{array}$ \\
\hline Medical institutions & 11,133 & 359,489 & 221,272 & 138,217 \\
\hline Hospitals & 1,409 & 115,447 & 78,299 & 37,148 \\
\hline Clinics & 9,724 & 244,042 & 142,973 & 101,069 \\
\hline Dental care institutions & 1,004 & 28,391 & 18,175 & 10,216 \\
\hline Pharmacies & 4,816 & 77,121 & 47,037 & 30,084 \\
\hline
\end{tabular}

TABLE 2: Distributions of the usage of Kampo drugs for sex, patient type, and age.

\begin{tabular}{lccccccccccccccc}
\hline \multirow{2}{*}{ Drug } & \multirow{2}{*}{ Total $^{1}$} & \multicolumn{2}{c}{ Sex $^{2}$} & \multicolumn{2}{c}{ Patient } & \multicolumn{1}{c}{ Age } \\
& & Male & Female & Inpatient & Outpatient & $0-9$ & $10-19$ & $20-29$ & $30-39$ & $40-49$ & $50-59$ & $60-69$ & $70-79$ & $80-89$ & $90-98$ \\
\hline \multirow{2}{*}{ All } & $67,095,008$ & $42.81 \%$ & $57.19 \%$ & $2.88 \%$ & $97.12 \%$ & $9.05 \%$ & $4.70 \%$ & $5.28 \%$ & $7.86 \%$ & $7.82 \%$ & $11.19 \%$ & $18.58 \%$ & $21.75 \%$ & $11.82 \%$ & $1.95 \%$ \\
Kampo & 898,797 & $33.09 \%$ & $66.91 \%$ & $7.01 \%$ & $92.99 \%$ & $1.57 \%$ & $1.76 \%$ & $6.89 \%$ & $9.80 \%$ & $10.69 \%$ & $9.85 \%$ & $17.55 \%$ & $23.81 \%$ & $15.49 \%$ & $2.59 \%$ \\
\hline
\end{tabular}

${ }^{1}$ Kampo drugs were perscribed for $100(89,897 / 67,095,0081)=1.34 \%$ patients of the total number of prescription.

${ }^{2}$ For male, Kampo drugs were prescribed for $100((89,897 \times 0.3309) /(67,095,008 \times 0.4281))=1.04 \%$ patients. For female $100((89,897 \times$ $0.6691) /(67,095,008 \times 0.5719))=1.57 \%$.

health insurance schemes, the national health insurance scheme, and the medical care system for the elderly in later stages of life. We elucidate the usage of Kampo drugs by examining MHLW's records from 2009.

We assessed detailed statements selected from a stratified random two-stage sampling process; insurance-covered medical care institutions and pharmacies formed the primary sampling unit; and detailed statements were the secondary sampling unit (Table 1). We analysed the retrieved data for age, sex, diagnostic name, use of drugs (designation and dosage), and the number of prescriptions dispensed to inpatients and outpatients.

\section{Results}

The MHLW approves of three categories of Kampo drugs for reimbursement under the National Health Insurance Program. These include Kampo formulation extracts (extracts based on Kampo preparation formulae), crude drugs (including powdered crude drugs), and crude drug preparations. Of the 382 categories of approved drugs covered by public health insurance, they are the 28th $(0.80 \%), 177$ th $(0.05 \%)$, and 238th $(0.02 \%)$ most-prescribed categories, respectively (numbers in parentheses indicate the quantity of Kampo drugs as a percentage of the total number of retrieved and prescribed drugs). In the following analyses, only drugs of the first category-the Kampo formulation extracts, which are largely provided as granules or powders of extracts from multiple crude drugs-were included. Hereafter, we will refer to Kampo formulation extracts as Kampo extracts.

Table 2 shows descriptive statistics for the usage of Kampo drugs in patients' sex, type, and age. Kampo drugs accounted for $1.34 \%$ of the total number of prescriptions $(1.04 \%$ and $1.57 \%$ for male and female patients, resp.), with Kampo prescription being more frequent in female patients. As with other drugs, Kampo drugs were more frequently prescribed for outpatients (92.99\%) than for inpatients (7.01\%); this, however, was not the case for all Kampo extracts. Details are provided in a later section. We also found that Kampo drugs were prescribed more frequently than other drugs for young (under 20 years old) and elderly (older than 70 years old) patients.

We also assessed the joint use of Kampo extracts and biomedical drugs and found that $92.2 \%$ of patients who had been prescribed Kampo extracts were coadministered biomedical drugs during single consultations. The large percentage of patients undergoing combinatorial treatment reflects the uniqueness of the Japan's medical license and health care system, according to which physicians are allowed to prescribe both Kampo extracts and biomedical drugs. Statistics for diseases where Kampo extracts were prescribed as treatment are available in Table 3 and based on ICD-10 categories.

As shown in Table 4, the disease class "XV: pregnancy, childbirth, and the puerperium" contained the highest percentage $(4.05 \%$ of the total number of patients in the class) of patients who were prescribed Kampo extracts; this may be because Kampo extracts are known to have fewer and relatively milder effects than biomedical drugs in pregnant and postpartum women. The next most frequent disease categories were "XIV: diseases of the genitourinary system" (3.08\%) and "XI: diseases of the digestive system" $(2.94 \%)$. Kampo extracts are, therefore, frequently prescribed to women and patients with digestive diseases. They were rarely used for diseases of the eye and adnexa (category VII; $0.09 \%$ ) and congenital malformations, deformations, and chromosomal abnormalities (category XVII; $0.21 \%$ ). None of the patients categorized into "XVI: certain conditions originating in the perinatal period" received prescriptions for Kampo extracts. 
TABLE 3: Disease classification based on the International Classification of Diseases (ICD) 10th revision for 2008.

\begin{tabular}{ll}
\hline Category & Description \\
\hline I & Certain infectious and parasitic diseases \\
III & Neoplasms \\
IV & Diseases of the blood and blood-forming organs and certain disorders involving the immune mechanism \\
V & Endocrine, nutritional, and metabolic diseases \\
VI & Mental and behavioural disorders \\
VII & Diseases of the nervous system \\
VIII & Diseases of the eye and adnexa \\
IX & Diseases of the ear and mastoid process \\
X & Diseases of the circulatory system \\
XI & Diseases of the respiratory system \\
XII & Diseases of the digestive system \\
XIII & Diseases of the skin and subcutaneous tissue \\
XIV & Diseases of the musculoskeletal system and connective tissue \\
XV & Diseases of the genitourinary system \\
XVI & Pregnancy, childbirth, and the puerperium \\
XVII & Certain conditions originating in the perinatal period \\
XVIII & Congenital malformations, deformations, and chromosomal abnormalities \\
XIX & Symptoms, signs, and abnormal clinical and laboratory findings, not elsewhere classified \\
\hline
\end{tabular}

http://apps.who.int/classifications/icd10/browse/2008/en.

TABLE 4: Ratios of Kampo extract use per ICD-10 category.

\begin{tabular}{|c|c|c|c|c|c|}
\hline \multirow{2}{*}{$\begin{array}{l}\text { ICD-10 category } \\
\text { I }\end{array}$} & \multicolumn{2}{|c|}{$\begin{array}{l}\text { Fraction of patients prescribed } \\
\text { Kampo drugs per ICD-10 } \\
\text { category }\end{array}$} & \multicolumn{2}{|c|}{ Fraction of patients per ICD-10 category } & \multirow{2}{*}{$\begin{array}{c}\text { Ratio of patients prescribed } \\
\text { Kampo drugs to total number of } \\
\text { patients } \\
1.39 \%\end{array}$} \\
\hline & 37,458 & $(4.17 \%)$ & $2,698,197$ & $(4.02 \%)$ & \\
\hline II & 34,688 & $(3.86 \%)$ & $2,811,687$ & $(4.19 \%)$ & $1.23 \%$ \\
\hline III & 2,933 & $(0.33 \%)$ & 243,543 & $(0.36 \%)$ & $1.20 \%$ \\
\hline IV & 81,481 & $(9.06 \%)$ & $6,020,386$ & $(8.97 \%)$ & $1.35 \%$ \\
\hline $\mathrm{V}$ & 50,770 & $(5.65 \%)$ & $3,171,006$ & $(4.72 \%)$ & $1.60 \%$ \\
\hline VI & 23,817 & $(2.65 \%)$ & $1,752,559$ & $(2.61 \%)$ & $1.36 \%$ \\
\hline VII & 5,836 & $(0.65 \%)$ & $6,865,056$ & $(10.23 \%)$ & $0.09 \%$ \\
\hline VIII & 3,671 & $(0.41 \%)$ & $1,142,209$ & $(1.70 \%)$ & $0.32 \%$ \\
\hline IX & 189,799 & (21.11\%) & $13,456,856$ & $(20.05 \%)$ & $1.41 \%$ \\
\hline $\mathrm{X}$ & 117,320 & (13.05\%) & $8,920,202$ & $(13.29 \%)$ & $1.32 \%$ \\
\hline XI & 110,688 & $(12.31 \%)$ & $3,761,886$ & $(5.61 \%)$ & $2.94 \%$ \\
\hline XII & 36,727 & $(4.08 \%)$ & $4,178,405$ & $(6.23 \%)$ & $0.88 \%$ \\
\hline XIII & 101,762 & $(11.32 \%)$ & $6,336,119$ & $(9.44 \%)$ & $1.61 \%$ \\
\hline XIV & 72,101 & $(8.02 \%)$ & $2,344,046$ & $(3.49 \%)$ & $3.08 \%$ \\
\hline $\mathrm{XV}$ & 10,501 & $(1.17 \%)$ & 259,084 & $(0.39 \%)$ & $4.05 \%$ \\
\hline XVI & 0 & $(0.00 \%)$ & 55,404 & $(0.08 \%)$ & $0.00 \%$ \\
\hline XVII & 336 & $(0.04 \%)$ & 161,658 & $(0.24 \%)$ & $0.21 \%$ \\
\hline XVIII & 7,459 & $(0.83 \%)$ & 777,263 & $(1.16 \%)$ & $0.96 \%$ \\
\hline XIX & 11,835 & $(1.32 \%)$ & $2,158,013$ & $(3.22 \%)$ & $0.55 \%$ \\
\hline Total & 899,182 & $(100.00 \%)$ & $67,113,579$ & $(100.00 \%)$ & $1.34 \%$ \\
\hline
\end{tabular}


Table 5 shows the top ten most-prescribed Kampo extracts. Kakkonto, tokishakuyakusan, and shoseiryuto were prescribed for younger patients. Shakuyakukanzoto, daikenchuto, and goshajinkigan, meanwhile, were prescribed for elderly patients. Daikenchuto and goshajinkigan were more often prescribed for males than for females; tokishakuyakusan and bofutsushosan, in turn, were mostly prescribed for female patients. As with other traditional medicines, Kampo medicine is generally used as primary care for outpatients. Nevertheless, daikenchuto was more frequently administered to inpatients than outpatients.

The most prescribed Kampo drug was shakuyakukanzoto, which is widely used in musculoskeletal disorders such as muscle cramps and lumbago or associated conditions, such as dysmenorrhea. Shakuyakukanzoto acts on not only skeletal [10] but also smooth muscles [11].

Some Kampo extracts were specifically used for certain diseases; bakumondoto, for instance, was most frequently prescribed for bronchitis or bronchial asthma. Other Kampo extracts were applied to a wide variety of disease categories: hochuekkito, for instance, is prescribed for diseases of the circulatory system (category IX), digestive diseases (category $\mathrm{XI}$ ), and conditions affecting the genitourinary system (category XIV), among others. Many Kampo extracts are coadministered with biomedical drugs. Kakkonto was prescribed with gargles and troches (13.23\%), compresses containing anti-inflammatory chemicals $(15.32 \%)$, or antipyretics $(5.75 \%$ (acetaminophen)). Goshajinkigan was often used with anticancer drugs $(20.24 \%)$.

\section{Discussion}

The Japanese health care system is unique in that Japan is the only country where traditional medicine is fully integrated alongside with modern medicine in daily practice. Currently, Japanese physicians, all trained in western medical schools, employ both biomedicine and traditional Japanese Kampo medicine in the clinic and university hospitals [5]. Kampo medicine, which originated in ancient China, had been Japan's primary health care system for over 1,500 years prior to the Meiji Restoration (1868-1912). In 1874, the government approved the Medical Care Law, which called for the adoption of the German model of health care and only legitimized western medical licenses. Early-twentiethcentury physicians, however, continued to work towards reinstating Kampo as an official part of Japan's health care system.

In 1967, the first four Kampo extracts were approved for reimbursement under the National Health Insurance system. At the time of publication, this number has increased to 148 Kampo formulation extracts, 241 crude drugs, and 5 crude drug preparations. The present study examined only Kampo extracts because most Kampo drugs are prescribed in this form. The government also established the Good Manufacturing Practice (GMP) law in 1987 to ensure that all Kampo products are of uniformly high quality. The pharmaceutical industry, too, focused on Kampo medicine, engaging in research and development of high quality Kampo extracts.
During the 52-year history of National Health Insurance in Japan, traditional medicine was also covered; as a result, both medicines coexist in one system [5].

However, the current status of traditional medicine's integration was largely unknown despite the MHLW's systematically collecting data on more than 60 million health care claims every year. We have analysed the use of Kampo medicine on the basis of these data.

We found that $1.34 \%$ of all patients were prescribed Kampo drugs. This appears small when contrasted with the fact that $70-80 \%$ of Japanese physicians prescribe Kampo drugs in their daily practice [5-9]. How can we explain this gap? Even though most physicians use Kampo drugs in daily practice, the proportion of patients who receive prescriptions for Kampo drugs is very small. Most physicians use only biomedicine and occasionally add Kampo drugs. Although we can speculate that the indications of Kampo drugs are very limited, Table 4 shows that all types of diseases are covered by Kampo treatment.

In Japan, most physicians are educated to be specialists. The Japanese government considers this to be problematic in an ageing society like Japan's. Elderly patients have multiple complaints at once, and general physicians are in demand in Japan. Our results show that indications of Kampo drugs are limited to one physician according to that physician's specialty, perhaps due to a western biomedical diagnosis. This is not in accordance with traditional use of Kampo drugs: Kampo medicine is a system similar to general medicine.

Until 2001, very few medical schools taught Kampo medicine. Most of physicians had not been educated in either general or Kampo medicine. In 2001, the Ministry of Education, Science, and Technology set new guidelines that incorporated Kampo education into the core curriculum of Japanese medical schools. Additionally, medical society features more and more the importance of general medicine. This may lead to a change in physicians' attitudes in the near future.

In the present study, we found that the use of Kampo extracts is relatively common in women with category XV health conditions. This may explain that why female patients are prescribed Kampo more frequently than are male patients; menopausal syndromes [12-15] and premenstrual syndrome are good indications of Kampo medicine and exclusively affect women.

The most frequently prescribed Kampo extract was shakuyakukanzoto, which is indicated for muscle cramp, lumbago, and abdominal pain. This drug is used as a muscle relaxant. Because it is so effective, many physicians use it as an alternative to analgesics. This Kampo extract is composed of 6 grams of peony root and 6 grams of liquorice root. Liquorice root contains glycyrrhizin and may cause pseudoaldosteronism, which can be very severe in rhabdomyolysis. Interestingly, liquorice root is included in $70 \%$ of the aforementioned 148 Kampo extracts. Physicians sometimes combine Kampo extracts, which increases the risk of pseudoaldosteronism [16]. To avoid this, proper education and drug information are necessary.

The use of Kampo has been modernized with western biomedicine. For example, the most commonly used Kampo 


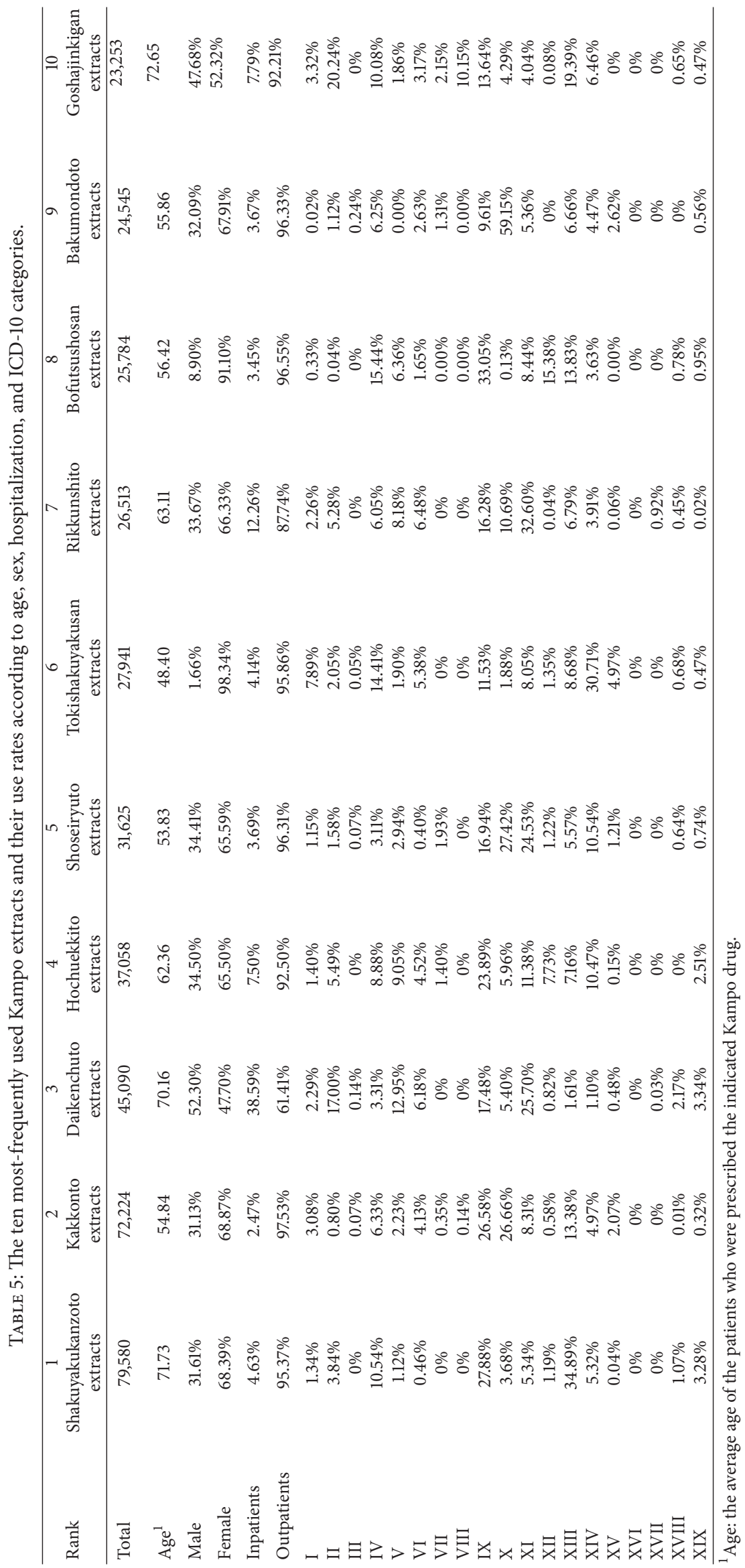


drug for digestive diseases was daikenchuto. In Jinkui Yaolue, an ancient textbook of the second century, daikenchuto, was indicated for abdominal pain caused by the cold. Currently in Japan, daikenchuto is used for prevention of postsurgical ileus. Yasunaga et al. also showed that daikenchuto improved postoperative ileus requiring long-tube decompression [17]. Because the mechanism of action is well understood [1820], many surgeons use daikenchuto routinely after operation; some hospitals have even adopted daikenchuto into their established clinical course. This is why daikenchuto is used more frequently in hospital settings than other Kampo extracts, which are generally used for outpatients in primary care settings.

Our data shows that $92.2 \%$ of patients prescribed Kampo extracts used them in combination with biomedical drugs. Goshajinkigan is one such Kampo extract that is often prescribed with anticancer drugs. It is frequently used to reduce numbness of the extremities, an adverse effect of anticancer drugs [21].

Another example is kakkonto, which is mainly prescribed for the treatment of the common cold. It was coadministered with gargles and troches. We also found that it was prescribed with antipyretics at a considerably high rate. In the view of Kampo medicine, such a combination is not ideal: one of the main effects of kakkonto is to raise the body temperature in order to eliminate high-temperature-sensitive viruses. If antipyretics are used in combination with kakkonto, the effect of kakkonto will be masked. It is possible that such misuse occurs because physicians ignored Kampo medicine theory and prescribed it in the light of westernized biomedicine.

There are only 2,420 board-certified Kampo doctors among the 280,000 licensed physicians of Japan. Historically, Kampo treatment was advised based on the patient's total body pattern. For effective Kampo treatment, drugs must be used in their traditional way. Additionally, although many physicians demand clinical evidence of Kampo medicine, most of the Kampo clinical studies have been performed based on western diagnoses [22].

Therefore, in order to prescribe Kampo extracts properly and avoid their misuse, education and clinical evidence based on traditional use of Kampo medicine should be established. In order to do so, standardized Kampo diagnosis is necessary. The WHO sets out to create an international classification of traditional medicine, and plans to incorporate it into the ICD-11 revision [2-4]. Mutual communication between traditional medicine and biomedicine specialists will thereupon be promoted and become more visible internationally. It is necessary to extend Kampo education in medical schools and to set up systematic continuous Kampo education after graduation. The safety and effectiveness of Kampo treatments are of utmost importance. Knowledge of Kampo diagnosis and proper use of Kampo drugs are, therefore, essential. This will ensure that future generations of Japanese physicians are able to truly integrate Kampo medicine into their practice.

\section{Conclusion}

We shed light on the prescription rate of Kampo drugs in Japan, analysing 67,113,579 health care claims records collected by the Ministry of Health, Labour and Welfare. The rate of Kampo prescription is still very low compared to the high rates of physicians' use of Kampo drugs. This gap may be caused by the Kampo drugs that were not used based on Kampo medicine theory, but by western biomedical diagnosis. Kampo drugs are more effective when they are used in the proper Kampo way, and proper education in this regard may be necessary.

\section{Acknowledgments}

This study was partially supported by Health and Labour Sciences Research Grants for Clinical Research and Research on Statistics and Information from the Ministry of Health, Labour and Welfare of Japan. The authors also thank the Social Statistics Division and International Classification and Information Management Office of Statistics and Information Department, Ministry of Health, Labour, and Welfare of Japan for providing health care claim records.

\section{References}

[1] International Classification of Diseases (ICD), World Health Organization, http://www.who.int/classifications/icd/en/.

[2] D. Normile, "WHO Shines a Light on Traditional Medicine," Science Insider, 2010, http://news.sciencemag.org/scienceinsider/2010/12/who-shines-a-light-on-traditional.html.

[3] L. Stafford, "WHO developing new traditional medicine classification," Herbal E Gram, vol. 8, no. 1, 2011, http://cms.herbalgram.org/heg/volume8/01January/WHOClassifiesTM.html?t= 1294841964.

[4] K. Watanabe, X. Zhang, and S.-H. Choi, "Asian medicine: a way to compare data," Nature, vol. 482, no. 7384, p. 162, 2012.

[5] H. Yamashita, H. Tsukayama, and C. Sugishita, "Popularity of complementary and alternative medicine in Japan: a telephone survey," Complementary Therapies in Medicine, vol. 10, no. 2, pp. 84-93, 2002.

[6] K. Fujiwara, J. Imanishi, S. Watanabe, K. Ozasa, and K. Sakurada, "Changes in attitudes of Japanese doctors toward complementary and alternative medicine comparison of surveys in 1999 and 2005 in Kyoto," Evidence-Based Complementary and Alternative Medicine, vol. 2011, Article ID 608921, 7 pages, 2011.

[7] K. Watanabe, K. Matsuura, P. Gao et al., "Traditional Japanese Kampo medicine: clinical research between modernity and traditional medicine - the state of research and methodological suggestions for the future," Evidence-Based Complementary and Alternative Medicine, vol. 2011, Article ID 513842, 19 pages, 2011.

[8] E. C. Moschik, C. Mercado, T. Yoshino, K. Matsuura, and K. Watanabe, "Usage and attitudes of physicians in Japan concerning traditional Japanese medicine (Kampo Medicine): a descriptive evaluation of a representative questionnairebased survey," Evidence-based Complementary and Alternative Medicine, vol. 2012, Article ID 139818, 13 pages, 2012.

[9] A. Ito, K. Munakata, Y. Imazu, and K. Watanabe, "First nationwide attitude survey of Japanese physicians on the use of traditional Japanese medicine (kampo) in cancer treatment," Evidence-Based Complementary and Alternative Medicine, vol. 2012, Article ID 957082, 8 pages, 2012.

[10] H. Kusunoki, K. Haruma, J. Hata et al., "Efficacy of Rikkunshito, a traditional Japanese medicine (Kampo), in treating functional 
dyspepsia," Internal Medicine, vol. 49, no. 20, pp. 2195-2202, 2010.

[11] F. Hinoshita, Y. Ogura, Y. Suzuki et al., "Effect of orally administered Shao-Yao-Gan-Cao-Tang (Shakuyaku-kanzo-to) on muscle cramps in maintenance hemodialysis patients: a preliminary study," American Journal of Chinese Medicine, vol. 31, no. 3, pp. 445-453, 2003.

[12] V. Scheid, T. Ward, W.-S. Cha, K. Watanabe, and X. Liao, "The treatment of menopausal symptoms by traditional East Asian medicines: review and perspectives," Maturitas, vol. 66, no. 2, pp. 111-130, 2010.

[13] G. A. Plotnikoff and K. Watanabe, "New insights on women's health from Japan,” Minnesota Physician, vol. 12, pp. 32-33, 2004.

[14] G. A. Plotnikoff, K. Watanabe, and F. Yashiro, "Kampo-from old wisdom comes new knowledge," Herbal Gram, vol. 78, pp. 4657, 2008.

[15] G. A. Plotnikoff, K. Watanabe, C. Torkelson, J. la Valleur, and D. M. Radosevich, “The TU-025 keishibukuryogan clinical trial for hot flash management in postmenopausal women: results and lessons for future research," Menopause, vol. 18, no. 8, pp. 886-892, 2011.

[16] H. Kinoshita, M. Okabayashi, M. Kaneko et al., "Shakuyakukanzo-to induces pseudoaldosteronism characterized by hypokalemia, rhabdomyolysis, metabolic alkalosis with respiratory compensation, and increased urinary cortisol levels," Journal of Alternative and Complementary Medicine, vol. 15, no. 4, pp. 439443, 2009.

[17] H. Yasunaga, H. Miyata, H. Horiguchi, K. Kuwabara, H. Hashimoto, and S. Matsuda, "Effect of the Japanese herbal kampo medicine Dai-kenchu-to on postoperative adhesive small bowel obstruction requiring long-tube decompression: a propensity score analysis," Evidence-based Complementary and Alternative Medicine, vol. 2011, Article ID 264289, 7 pages, 2011.

[18] T. Kono, T. Kanematsu, and M. Kitajima, "Exodus of Kampo, traditional Japanese medicine, from the complementary and alternative medicines: is it time yet?" Surgery, vol. 146, no. 5, pp. 837-840, 2009.

[19] P. Murata, Y. Kase, A. Ishige, H. Sasaki, S. Kurosawa, and T. Nakamura, "The herbal medicine Dai-kenchu-to and one of its active components [6]-shogaol increase intestinal blood flow in rats," Life Sciences, vol. 70, no. 17, pp. 2061-2070, 2002.

[20] C. Shibata, I. Sasaki, H. Naito, T. Ueno, and S. Matsuno, “The herbal medicine Dai-Kenchu-Tou stimulates upper gut motility through cholinergic and 5-hydroxytryptamine 3 receptors in conscious dogs," Surgery, vol. 126, no. 5, pp. 918-924, 1999.

[21] T. Kono, N. Mamiya, N. Chisato et al., "Efficacy of goshajinkigan for peripheral neurotoxicity of oxaliplatin in patients with advanced or recurrent colorectal cancer," Evidence-based Complementary and Alternative Medicine, vol. 2011, Article ID 418481, 8 pages, 2011.

[22] Evidence Report of Kampo Treatment by Japan Society for Oriental Medicine, http://www.jsom.or.jp/medical/ebm/ ere/index.html. 


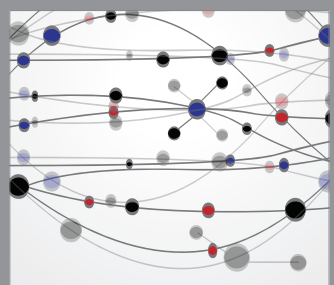

The Scientific World Journal
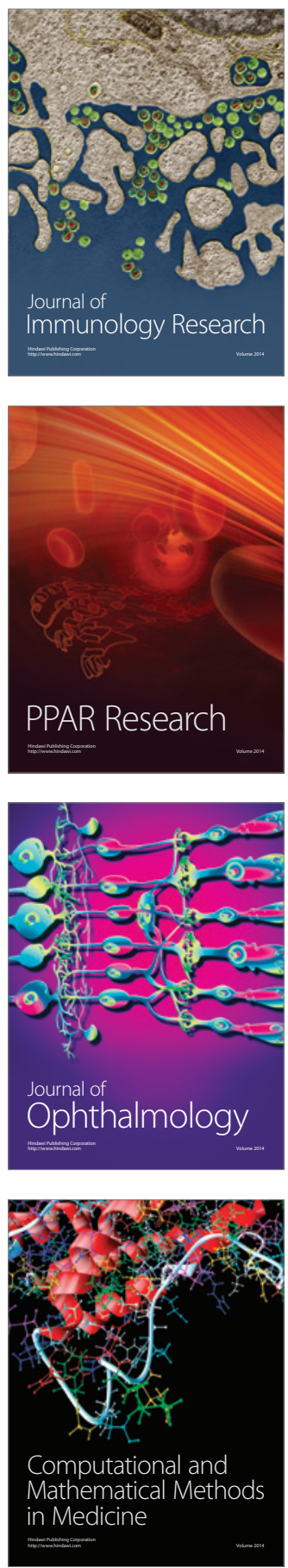

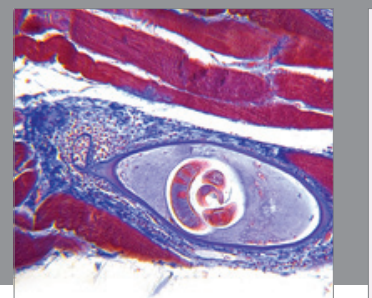

Gastroenterology

Research and Practice
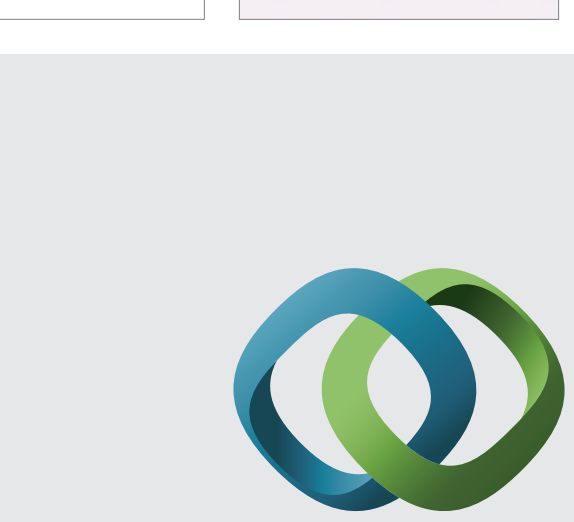

\section{Hindawi}

Submit your manuscripts at

http://www.hindawi.com
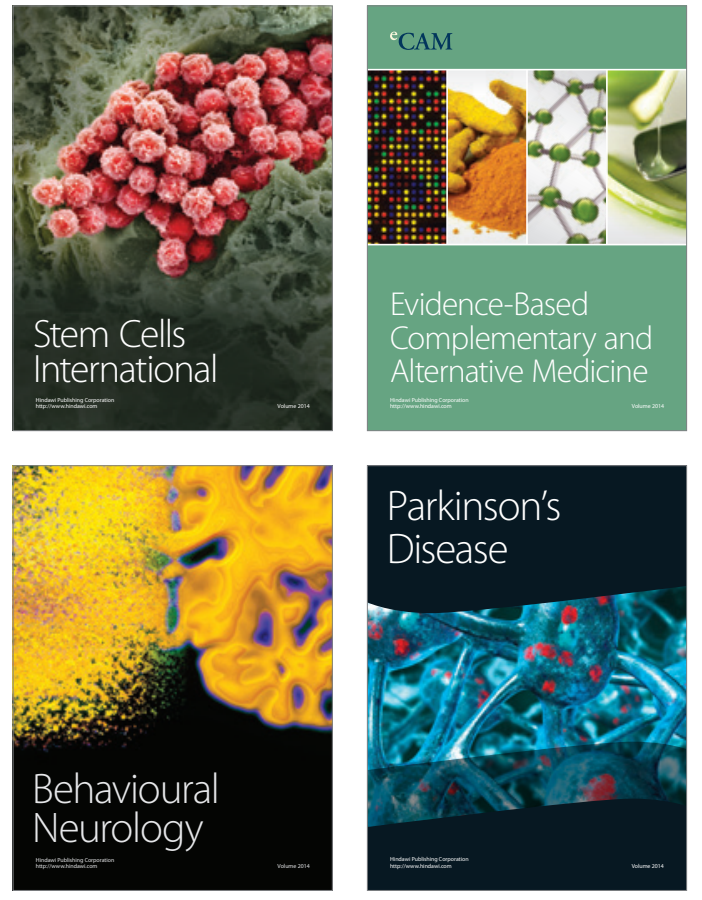
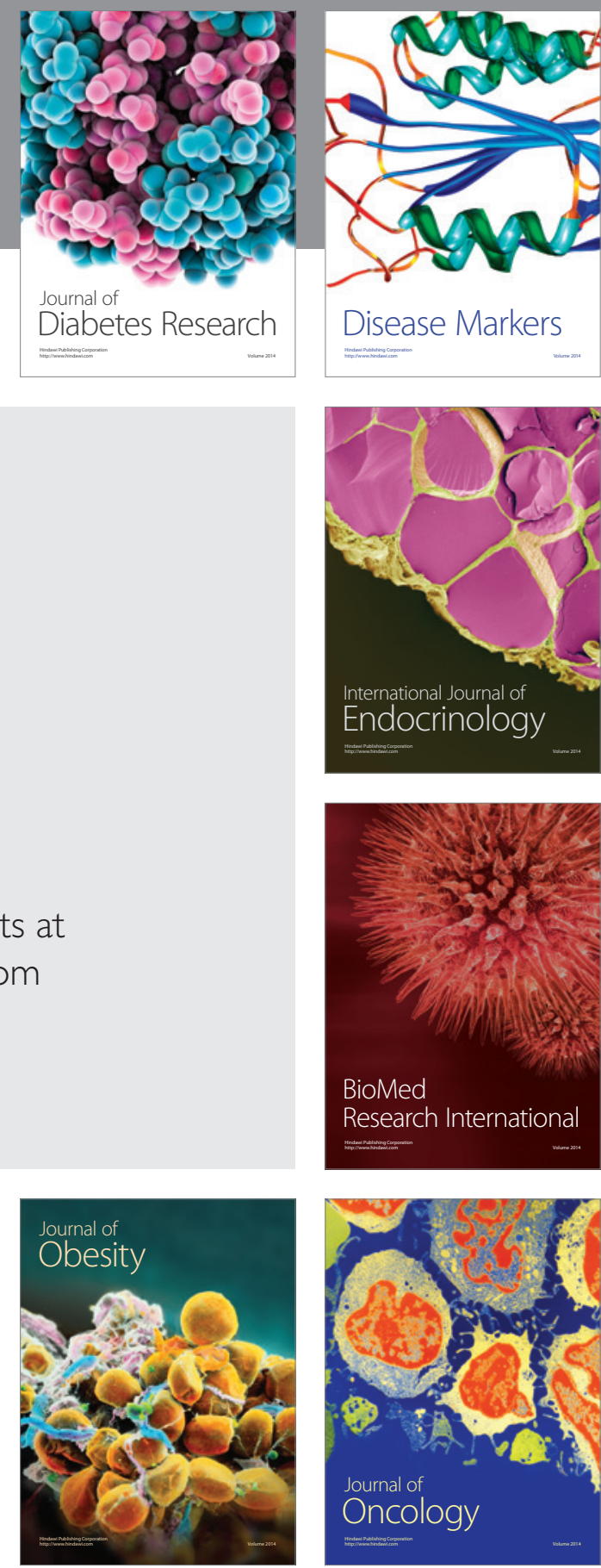

Disease Markers
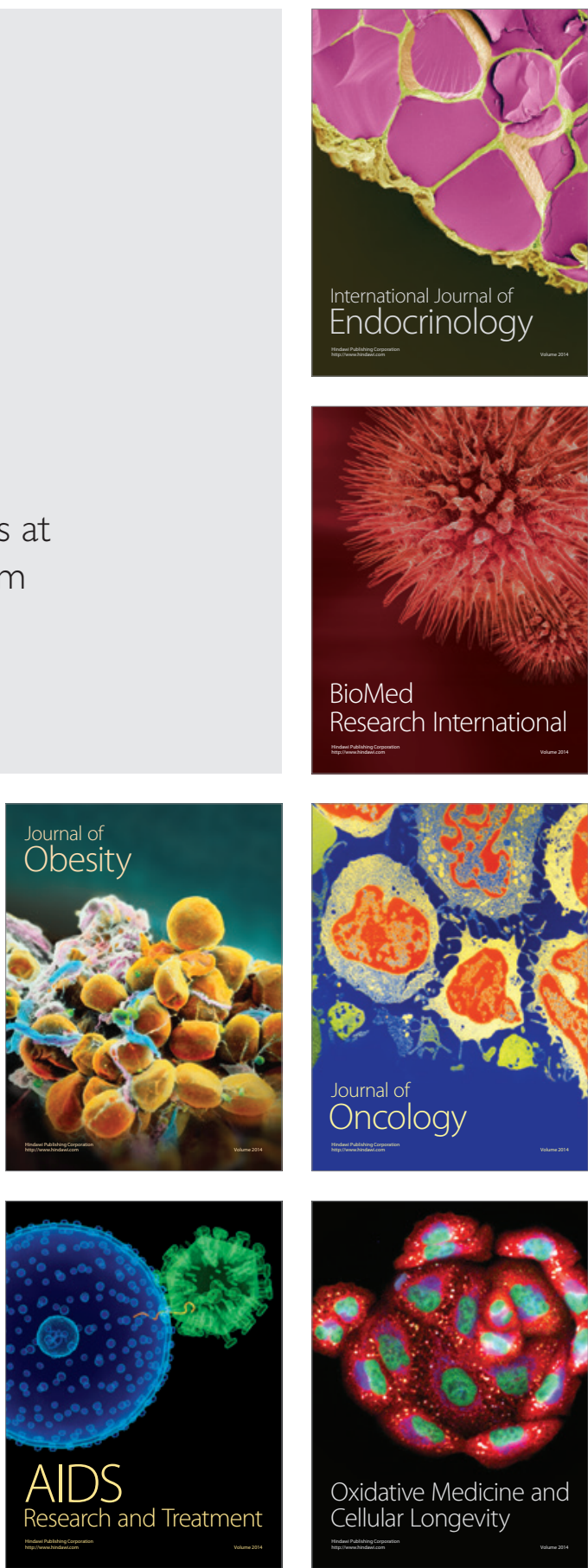